\title{
Understanding Pathologic Myopia
}

\author{
Kyoko Ohno-Matsui and Jost B. Jonas
}

\section{Key Points}

- Pathologic myopia is defined by the presence of posterior staphylomas and/or the presence of myopic chorioretinal atrophy equal to or more serious than diffuse atrophy.

- Myopic CNV is the most frequent cause of central vision loss.

- Ultra wide-field OCT is a useful tool to detect posterior staphylomas.

- Anti-VEGF therapies have greatly improved the prognosis of myopic CNV.

- Vitreoretinal surgeries for myopic macular retinoschisis are useful.

\subsection{Introduction}

Pathologic myopia (PM) is a major cause of blindness in the world, especially in East Asian countries [1-5]. The cause of blindness in patients with PM includes myopic maculopathy with or without posterior staphyloma, myopic macular retinoschisis, and glaucoma or glaucoma-like optic neuropathy. In this chapter, the lesions of myopic fundus complications including posterior staphylomas are described.

K. Ohno-Matsui $(\bowtie)$

Department of Ophthalmology and Visual Science, Tokyo Medical and Dental University,

Tokyo, Japan

e-mail: k.ohno.oph@tmd.ac.jp

\section{J. B. Jonas}

Department of Ophthalmology, University of Heidelberg, Heidelberg, Germany 


\subsection{Definition of Pathologic Myopia}

The terms "pathologic myopia," "high myopia," and "axial myopia" have long been used in a parallel manner in the literature. According to recent discussions, the term "high myopia" simply describes the status of a "high degree of myopia" and should be defined by a cut-off value of myopic refractive error. The term "axial myopia" may be used to describe the situation with an axial elongation as cause for the myopic refractive error. It is in contrast to the term refractive myopia, which is used for eyes with an abnormally high refractive power of their optical media. The term "pathologic myopia" describes the situation of pathologic consequences of a myopic axial elongation. According to a recent consensus article by Ohno-Matsui et al. [6], pathologic myopia was defined by myopic chorioretinal atrophy equal to or more serious than diffuse atrophy (by META-PM study group classification [7]) and/or the presence of posterior staphylomas.

\subsection{Posterior Staphyloma}

Posterior staphyloma has been considered a hallmark lesion of pathologic myopia. While axial elongation may primarily start in the equatorial and retro-equatorial region with secondary changes taking place at the posterior fundus, posterior staphylomas occur in the posterior segment of the eye and can be associated with, or lead to, vision-threatening complications in the macula as part of a myopic maculopathy [7-11] and myopic optic neuropathy/glaucoma $[12,13]$.

\subsubsection{Definition of Staphyloma by Spaide (Fig. 9.1)}

A posterior staphyloma is an outpouching of a circumscribed area of the posterior fundus, where the radius of curvature is less than the curvature radius of the surrounding eye wall [14].

\subsubsection{Detection of Posterior Staphyloma}

Moriyama et al. recently applied three-dimensional magnetic resonance imaging (3D-MRI) to analyze the shape of the entire eye from the corneal surface to the posterior pole including even large posterior staphylomas (Fig. 9.2) [15-17]. The technique allowed visualizing a staphyloma from any angle. The advantage of 3D-MRI of visualizing the shape of the whole eye including the anterior ocular segment is combined with its disadvantage of not being feasible as a screening technique. Instead, a new prototype of a wide-field swept-source optical coherence tomographic (OCT) system has been developed, which uses not only one but multiple scan lines and which generates scan maps allowing a three-dimensional 

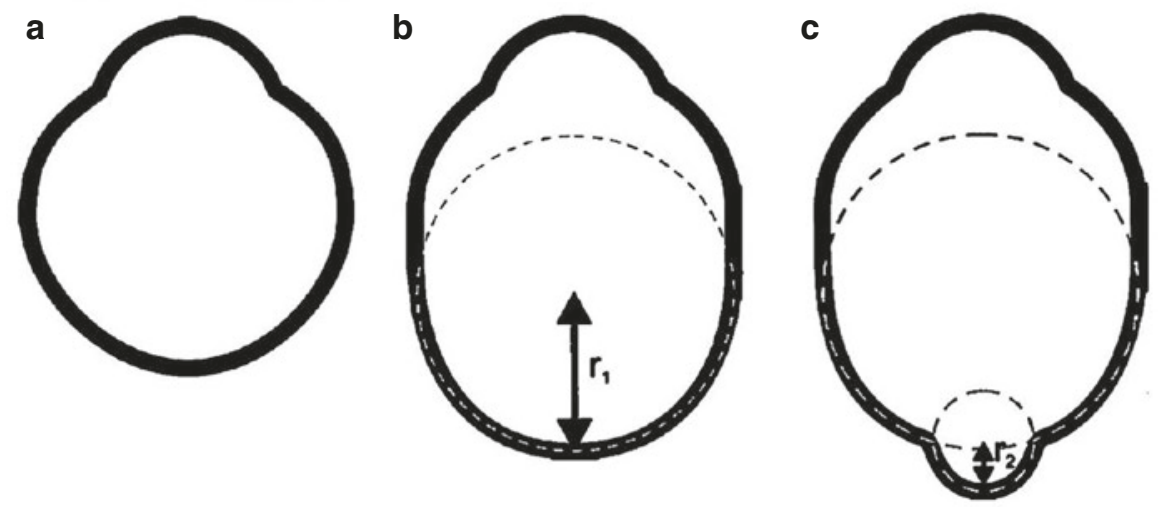

Fig. 9.1 Definition of posterior staphyloma (cited from the textbook Pathologic Myopia [14]). (a) Normal eye shape. (b) Axial expansion occurring in the equatorial region that does not induce any altered curvature in the posterior aspect of the eye. This eye would have axial myopia but no staphyloma. (c) Posterior staphyloma. A second curvature occurs in the posterior portion of the eye, and this second curvature has a small radius $\left(r_{2}\right)$ than the surrounding eye wall $\left(r_{1}\right)$. This secondary curve is a staphyloma. (This figure does not take into account changes in scleral thickness)

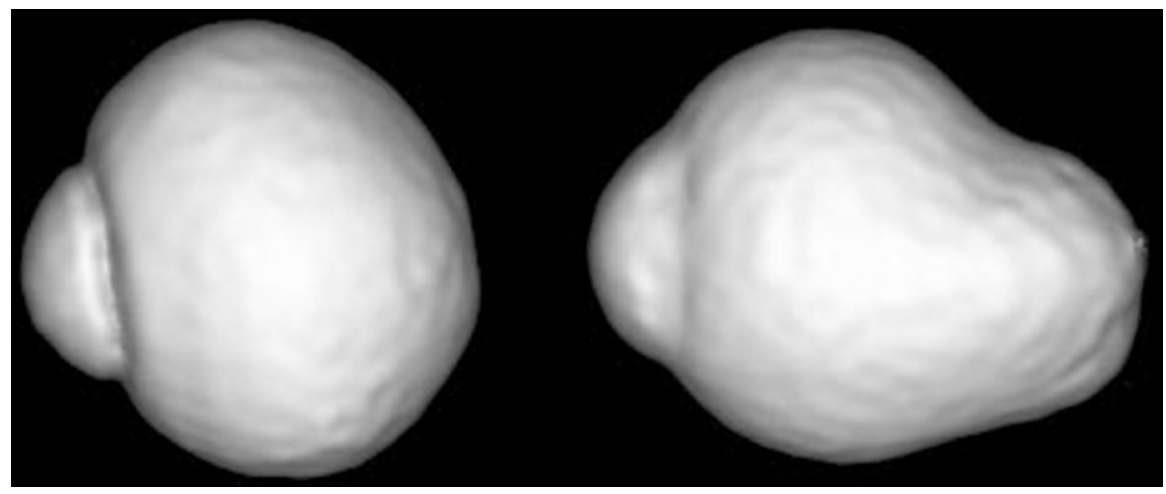

Fig. 9.2 Three-dimensional magnetic resonance images of eyes with emmetropia and posterior staphyloma. The shape of the emmetropic eye (left) is almost spherical and symmetrical, while the eye with a posterior staphyloma shows an outpouching of its posterior segment

reconstruction of posterior staphylomas in a region of interest of $23 \times 20 \mathrm{~mm}$ with a depth of $5 \mathrm{~mm}$. Applying a wide-field OCT (WF-OCT), Shinohara et al. [18] showed that WF-OCT could provide tomographic images of posterior staphylomas in a resolution and size unachievable up to that time, and that WF-OCT might replace 3D-MRI in examining posterior staphylomas. Upon WF-OCT, the edges of the staphylomas showed consistent features, consisting of a gradual thinning of the choroid from the periphery toward the staphyloma edge and a gradual rethickening of the choroid from the staphyloma edge in direction to the posterior pole (Fig. 9.3). 

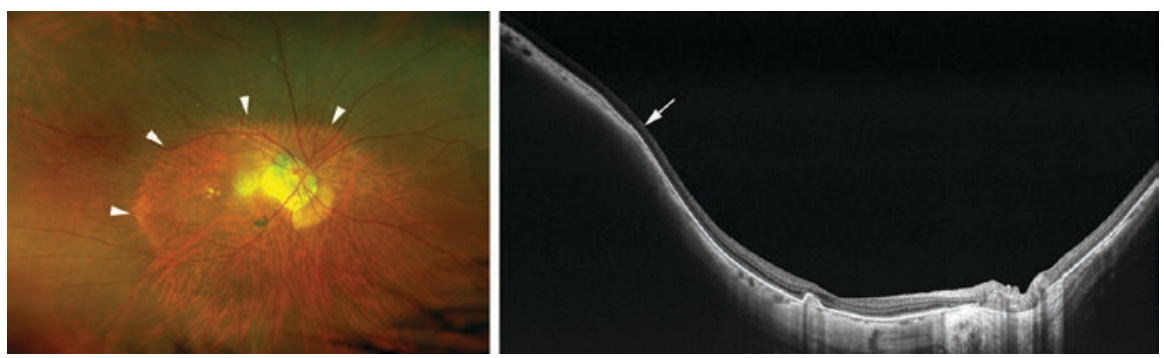

Fig. 9.3 Swept-source wide-field optical coherence tomographic (OCT) image of a posterior staphyloma. (Left) Right fundus shows a wide staphyloma (arrowheads). (Right) The wide-field OCT image shows the staphyloma edge (arrow) with a gradual thinning of the choroid from the periphery toward the staphyloma edge and a gradual re-thickening of the choroid toward the posterior pole. The white arrow indicates the change in the curvature radius of the sclera at the staphyloma edge

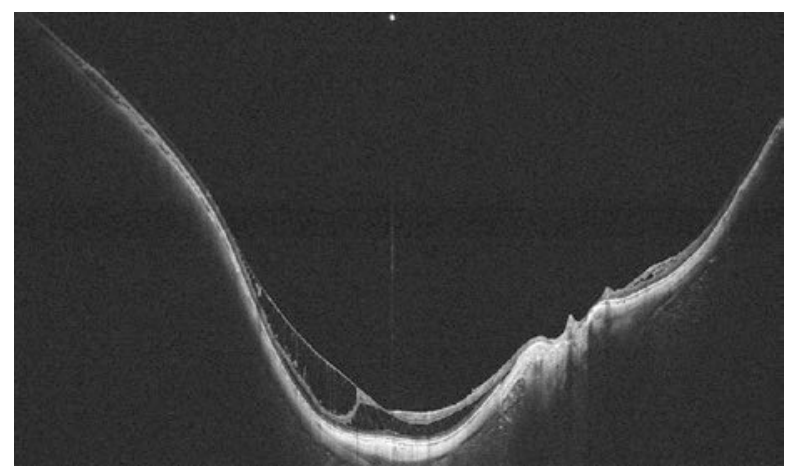

Fig. 9.4 Spatial relationship between myopic macular retinoschisis and posterior staphyloma. In this swept-source wide-field optical coherence tomographic image, the outer and inner retinoschisis is restricted to the area of the posterior staphyloma. It may suggest that the inner retinal structures as compared to the outer retinal structures were not flexible enough to follow the elongated circumference of Bruch's membrane and sclera

An additional advantage of the swept-source WF-OCT technology was the relatively large depth of focus so that structures from the posterior vitreous to the sclera could be imaged in the same image. It allowed the analysis of relationships between vitreoretinal abnormalities and other lesions in the inner retinal layers, such as myopic macular retinoschisis and posterior staphylomas as reported by Shinohara et al. [19] (Fig. 9.4).

\subsubsection{Classification (Ohno-Matsui's Modified Classification,} Fig. 9.3)

Based upon and modifying Curtin's [20] classical categorization of posterior staphylomas, with Types I-V as primary staphylomas and Types VI-X as compound staphylomas, Ohno-Matsui [17] used 3D-MRI and wide-field fundus 

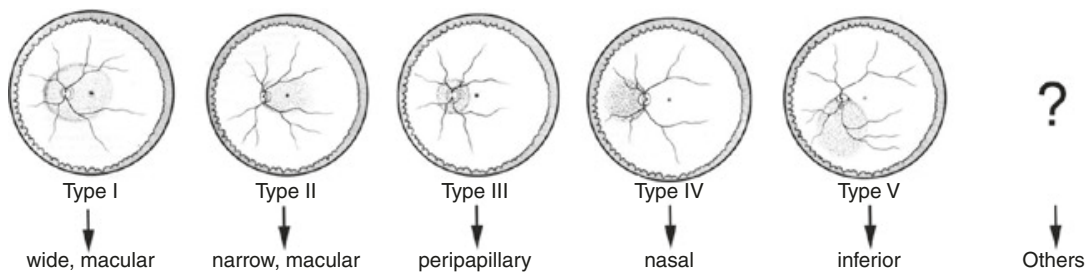

Fig. 9.5 Classification staphyloma (cited from [17] with permission). New classification of posterior staphyloma according to its location and extent. The staphyloma type is renamed according to its location and distribution. Type I $\rightarrow$ wide, macular staphyloma, Type II $\rightarrow$ narrow, macular staphyloma, Type III $\rightarrow$ peripapillary staphyloma, Type IV $\rightarrow$ nasal staphyloma, Type V $\rightarrow$ inferior staphyloma, others $\rightarrow$ staphylomas other than Types I-V

imaging to reclassify staphylomas into six types: the wide macular type, the narrow macular type, the peripapillary type, the nasal type, the inferior type, and others (Fig. 9.5).

\subsection{Fundus Complications of Pathologic Myopia}

\subsubsection{Myopic Chorioretinal Atrophy (META-PM Study, Table 9.1)}

In the META-PM classification [7], myopic maculopathy lesions have been categorized into five categories from "no myopic retinal lesions" (Category 0), "tessellated fundus only" (Category 1; Fig. 9.6a), "diffuse chorioretinal atrophy" (Category 2; Fig. 9.6b), "patchy chorioretinal atrophy" (Category 3; Fig. 9.6c), to "macular atrophy" (Category 4; Fig. 9.6d). These categories were defined based on long-term clinical observations that showed the progression patterns and associated factors of the development of myopic choroidal neovascularization (CNV) for each stage. Three additional features were added to these categories and were included as "plus signs": (1) lacquer cracks (Fig. 9.6e) and (2) myopic CNV (Fig. 9.6f) (Table 9.1). Since a Fuchs' spot represented a scarred form of myopic CNV, Fuchs' spots were categorized under the term of myopic CNV. The reason for separately listing the "plus signs" was that all three lesions have been shown to be strongly associated with central vision loss; however, they did not fit into any particular category and might develop from, or coexist, in eyes with any

Table 9.1 Myopic Chorioretinal Atrophy Classification (META-PM Study)

\begin{tabular}{l|l|l}
\hline & & Plus lesions \\
\hline Category 0 & Normal fundus & \multirow{2}{*}{ Myopic CNV lacquer cracks } \\
\hline Category 1 & Tesselated fundus & \\
\cline { 1 - 2 } Category 2 & Diffuse chorioretinal atrophy & \\
\cline { 1 - 2 } Category 3 & Patchy chorioretinal atrophy & \\
\cline { 1 - 2 } Category 4 & Macular atrophy & \\
\cline { 1 - 2 } & &
\end{tabular}




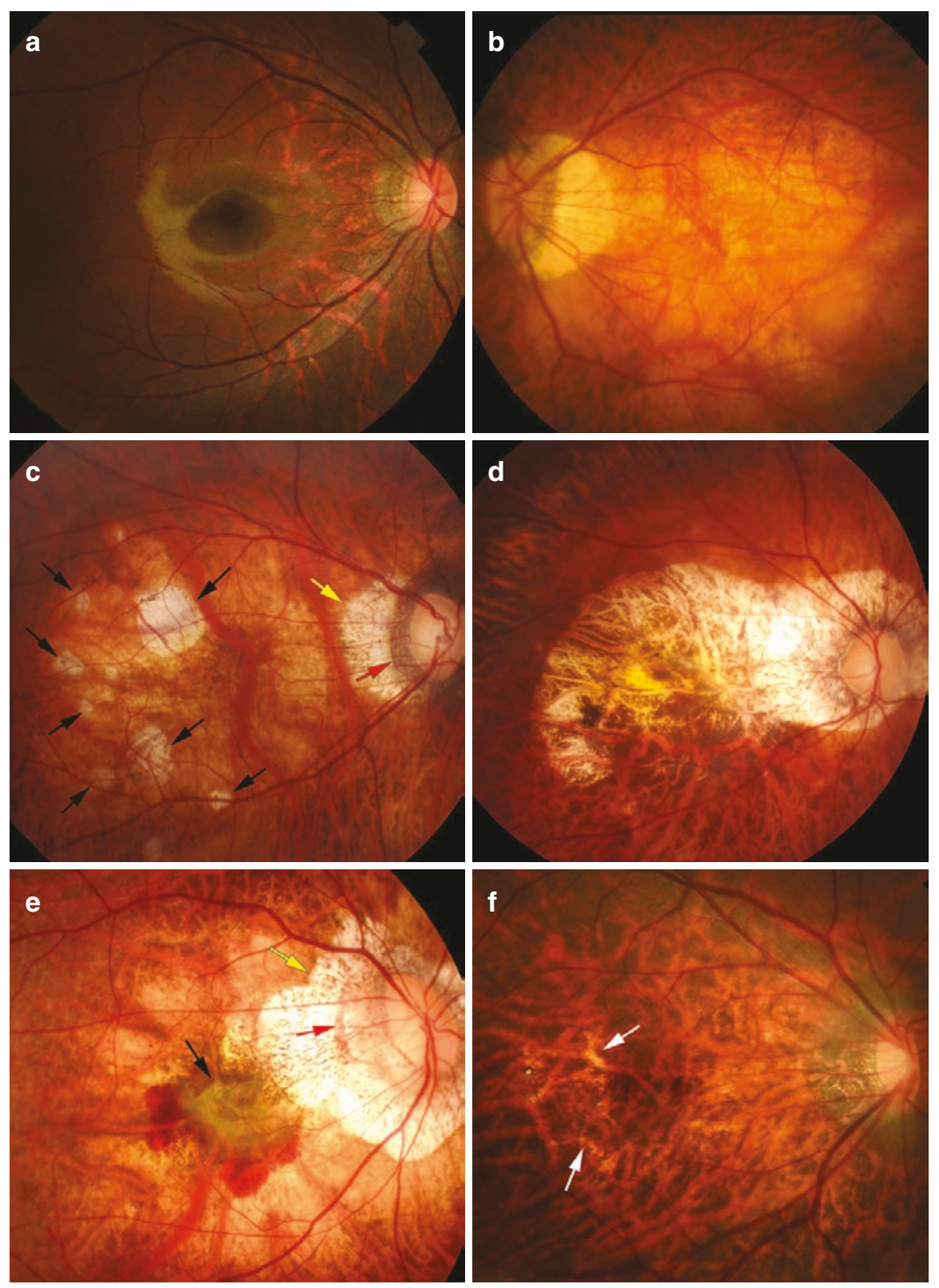

Fig. 9.6 Myopic maculopathy. (a) Fundus tessellation temporal to the optic disc. (b) Diffuse chorioretinal atrophy. Ill-defined, yellowish atrophy is seen in the posterior fundus. (c) Patchy chorioretinal atrophy. Within the area of diffuse atrophy, multiple areas of well-defined, whitish atrophic lesions are seen (arrows); yellow arrows: parapapillary gamma zone; red arrow: parapapillary delta zone. (d) Macular atrophy, several years after the formation of myopic choroidal neovascularization (CNV), a well-defined atrophic lesion is seen in the macular area. (e) Myopic CNV. Subretinal hemorrhage is seen around the CNV (arrow); yellow arrows: parapapillary gamma zone; red arrow: parapapillary delta zone. (f) Lacquer cracks. Multiple yellowish linear lesions are seen (arrows) 
of the myopic maculopathy categories described above. Based on this classification, pathologic myopia has now been defined as myopic maculopathy Category 2 or above, or the presence of "plus" sign or posterior staphyloma [7, 21].

\subsubsection{Diffuse Chorioretinal Atrophy (Category 2)}

Diffuse chorioretinal atrophy is characterized by a yellowish white appearance of the posterior pole. The region of the diffuse atrophy may extend from a restricted area around the optic disc and a part of the macula to the entire posterior pole. The atrophy generally first appears around the optic disc, often increases with age, and finally covers the entire area within a staphyloma if a staphyloma is present. Both older age and longer axial length have been described as risk factors for the development of diffuse atrophy [9]. Marked thinning of the choroidal layer in the area of diffuse atrophy can be detected upon OCT, with occasional large choroidal vessels remaining.

\subsubsection{Patchy Chorioretinal Atrophy (Category 3)}

Patchy chorioretinal atrophy appears as well-defined, grayish white lesion(s) in the macular area or around the optic disc. Upon OCT, the area of patchy atrophy is characterized by the absence of the entire choroid and the RPE as well as of the outer retina. Hyper-transmission through the underlying sclera can be seen upon OCT. Using swept-source OCT, Ohno-Matsui et al. [22] showed that patchy atrophy was not simply a chorioretinal atrophy but was combined with a defect in Bruch's membrane (BM).

\subsubsection{Lacquer Cracks (Plus Sign)}

Lacquer cracks appear as yellowish linear lesions in the macula. Lacquer cracks have been considered to represent breaks in BM [9, 23-25]. Progression patterns of lacquer cracks include an increased number, elongation, and progression to patchy atrophy $[8,26]$.

Detection of lacquer cracks can sometimes be difficult especially in eyes with diffuse atrophy. Assessments of hyper-fluorescence by fluorescein angiography and hypo-fluorescence upon indocyanine green angiography have been useful for the diagnosis. Other linear lesions due to pathologic myopia with a similar hypofluorescence upon by indocyanine green angiography, such as myopic stretch lines, have to be differentiated [27].

Due to their small width, linear defects in BM as the base of lacquer cracks are difficult to be directly detected. Instead, hyper-reflective lines of the choroidal and scleral tissue layers on the OCT images indirectly indicate the linear BM defect by an optical window effect (Fig. 9.7). The recently developed imaging technique of OCT angiography may be useful for detecting a rupture of the choriocapillaris in 

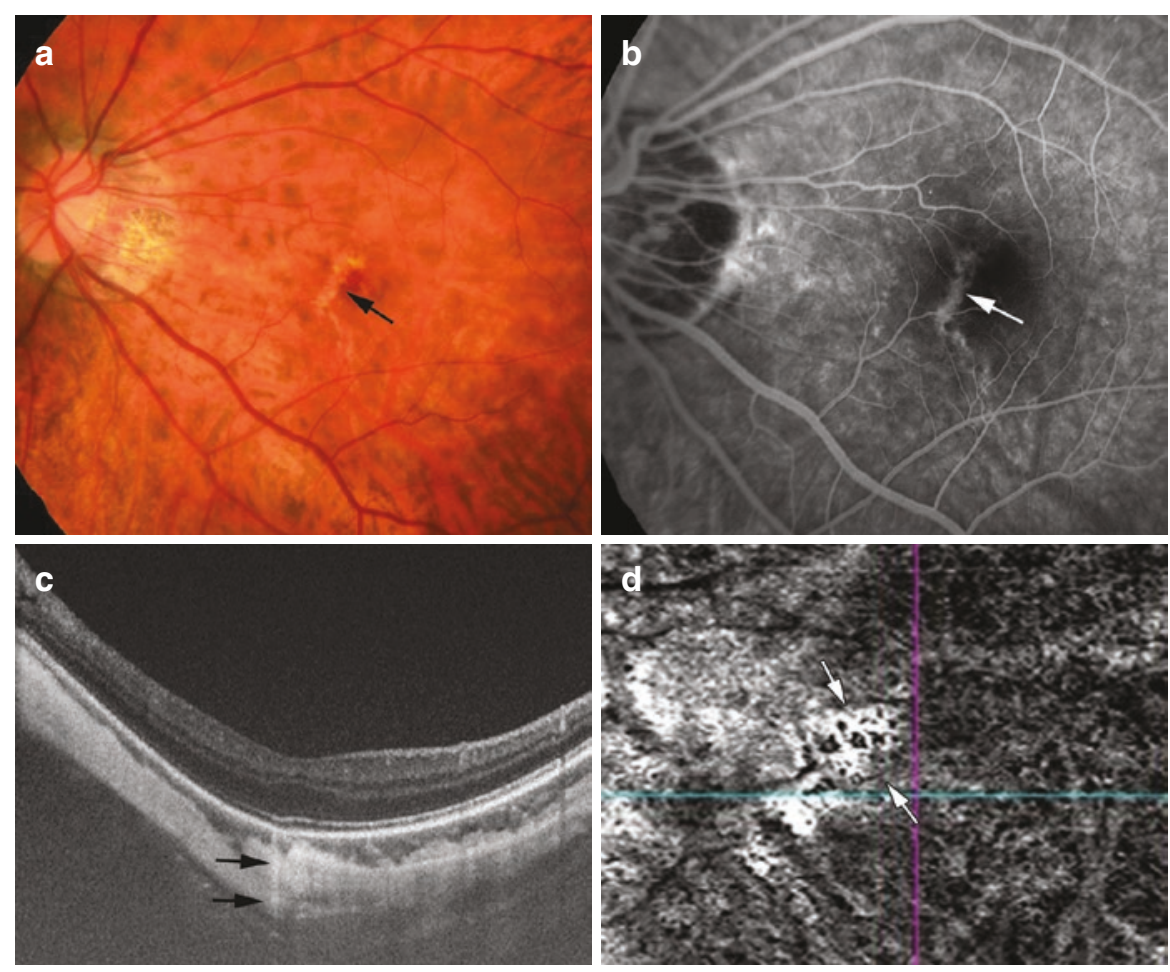

Fig. 9.7 Multimodal imaging of lacquer cracks. (a) Left fundus shows lacquer crack as yellowish linear lesion (arrow). (b) Fluorescein angiogram shows linear hyper-fluorescence at the site of lacquer crack (arrow). (c) Optical coherent tomographic image shows deep penetration of light (arrows) at the site of lacquer cracks. (d) OCT angiography shows a defect of choriocapillaris (arrows)

the area of lacquer cracks, again indirectly indicating a defect in BM [28]. Since a subretinal bleeding can often be observed at the onset of the development of lacquer cracks [29-31] (Fig. 9.8), it is important to exclude the presence of a myopic CNV in cases of subretinal bleeding in the region of lacquer cracks.

\subsubsection{Myopic CNV and CNV-Related Macular Atrophy}

Myopic CNV is a major sight threatening complication of pathologic myopia. It is the most common cause of $\mathrm{CNV}$ in individuals younger than 50 years, and it is the second most common cause of CNV overall [25, 32]. Myopic CNV is a Type II $\mathrm{CNV}$ and shows a clear hyper-fluorescence by fluorescein angiography. Anti-VEGF therapy is the first-line treatment for myopic CNV, as shown by the RADIANCE study [33] and the MYRROR study [34]. 

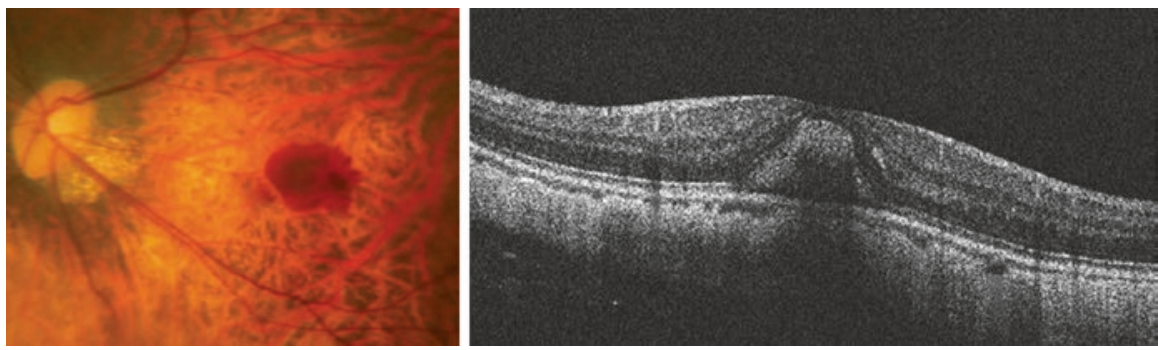

Fig. 9.8 Subretinal bleeding due to new lacquer crack formation. Left fundus shows subretinal bleeding. There are some projections along the temporal border of the hemorrhage. Optical coherent tomographic image shows subretinal hemorrhage. Serous retinal detachment or macular edema is not seen
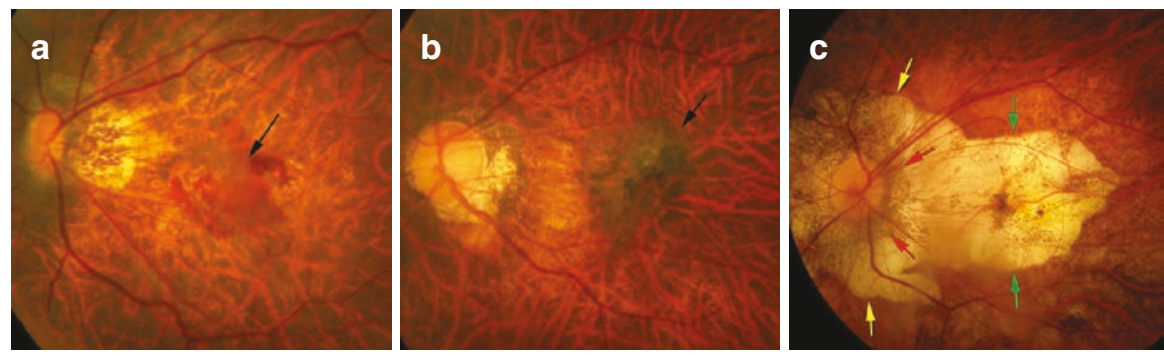

Fig. 9.9 Fundus photographs showing various phases of myopic CNV in different eyes. (a) Active phase. Subretinal bleeding is observed around the grayish CNV (black arrow). (b) Scar phase with pigmented scarred CNV (black arrow). (c) Atrophic phase at about 10 years after the first occurrence of a CNV; a well-defined macular atrophy (green arrows) appears as a macular Bruch's membrane defect, merging with a large parapapillary gamma zone (yellow arrows) around a parapapillary delta zone (red arrows)

In the long-term, both in treated eyes and in eyes exposed to the natural course of the disorder, macular atrophy develops around the scarred CNV and impairs central vision (Fig. 9.9). Swept-source OCT showed that CNV-related macular atrophy was not simply a chorioretinal atrophy but was BM hole [35], like patchy chorioretinal atrophy.

As shown upon OCT-angiography, the CNV maintains its blood flow even when the CNV transforms into the scar phase, including the area of CNV-related macular atrophy (Fig. 9.10). Louzada et al. [36] and Giuffre et al. [37] reported that blood vessels originating from the sclera were found at the site of myopic CNVs. Recently Ishida et al. [38] reported that the blood vessels of myopic CNVs were continuous to scleral branches of short posterior ciliary arteries (Fig. 9.11). 


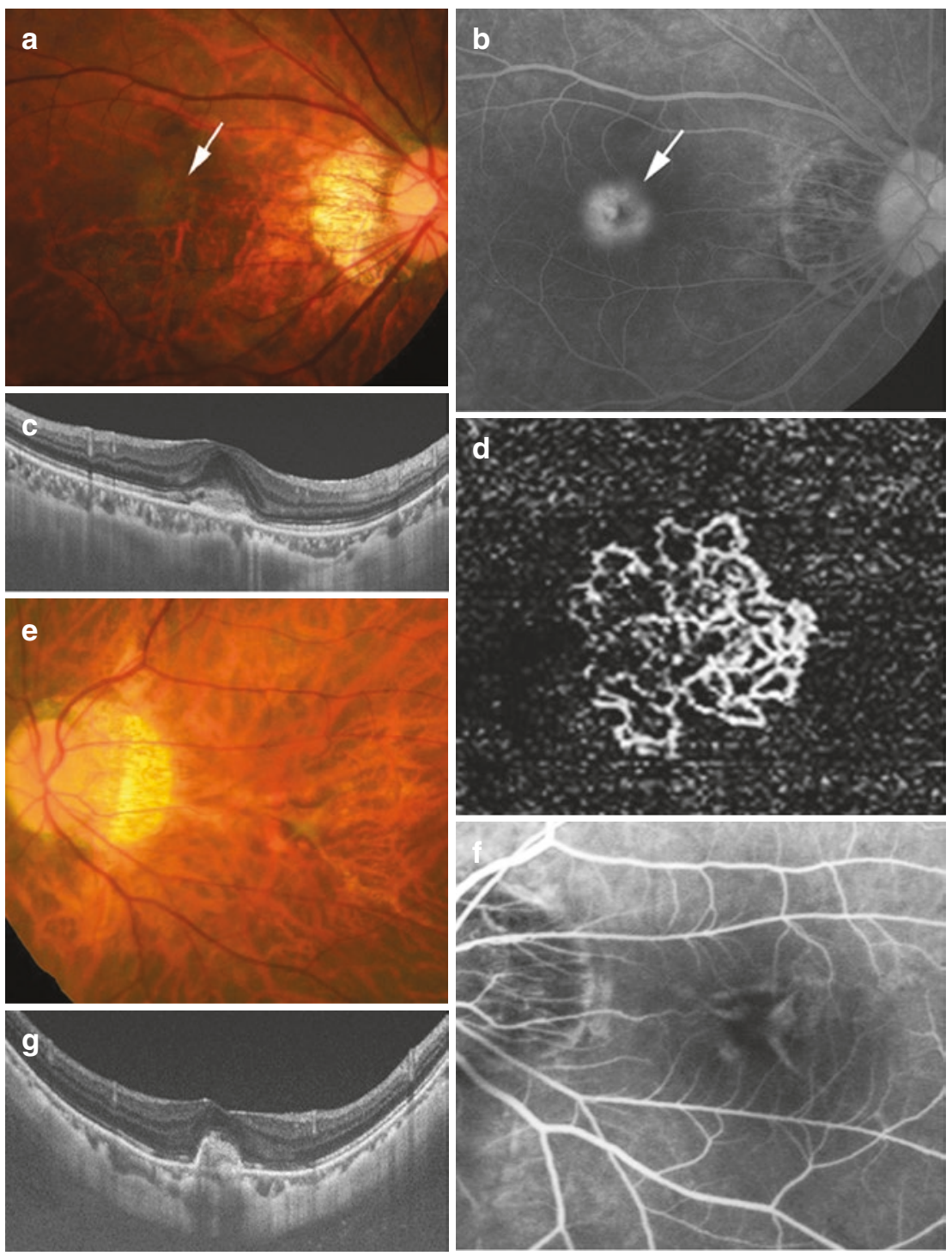

Fig. 9.10 Detection of choroidal neovascularization $(\mathrm{CNV})$ with blood flow in all of the three phases of myopic CNV by optical coherent tomographic angiography (OCTA) [38]. (a-d) Active phase. Right fundus shows a grayish CNV (arrow in a). Fluorescein angiography (FA) shows hyper-fluorescence at the CNV (arrow in b). (c) Type II CNV is observed as subretinal tissue with fuzzy border by OCT. (d) OCTA shows newly formed vessels. (e-h) Scar phase. (e) Left fundus shows irregular shape of CNV. (f) FA shows tissue staining of irregular shape of CNV. (g) OCT shows subretinal CNV with sharp margins. (h) OCTA shows spiky shaped CNV. (i-l) Atrophic phase. CNV-related macular atrophy (margined by arrowheads) is seen around the remnants of a CNV (arrow in i). Fundus autofluorescence shows clear hypo-fluorescence in the area of the macular atrophy around the CNV (arrow in j). OCT shows subretinal CNV (arrow in k) surrounded by a large defect of Bruch's membrane. The inner retina directly sits on the sclera in the area of macular atrophy. The choroid is barely seen in the area of the macular atrophy. (I) OCTA shows CNV with blood flow. Large blood vessels appear to be connected to the CNV 

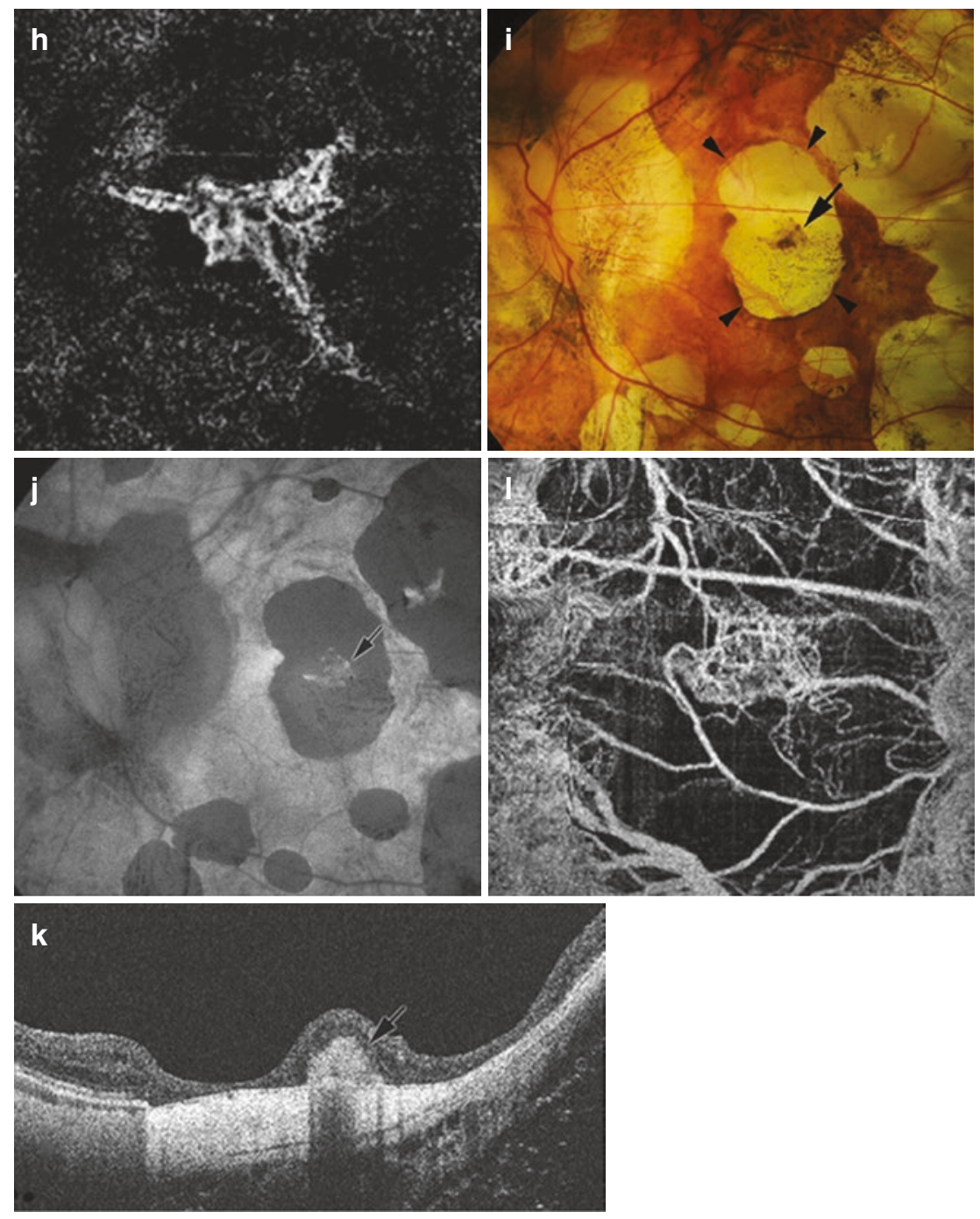

Fig. 9.10 (continued)

\subsection{Myopic Macular Retinoschisis}

Using optical coherence tomography, Takano and Kishi first demonstrated a foveal retinal detachment and retinoschisis in severely myopic eyes with posterior staphylomas [39]. Panozzo and Mercanti proposed the term "myopic traction maculopathy (MTM)" to encompass various findings characterized by a traction as visualized by OCT in highly myopic eyes [40]. Myopic traction maculopathy, 

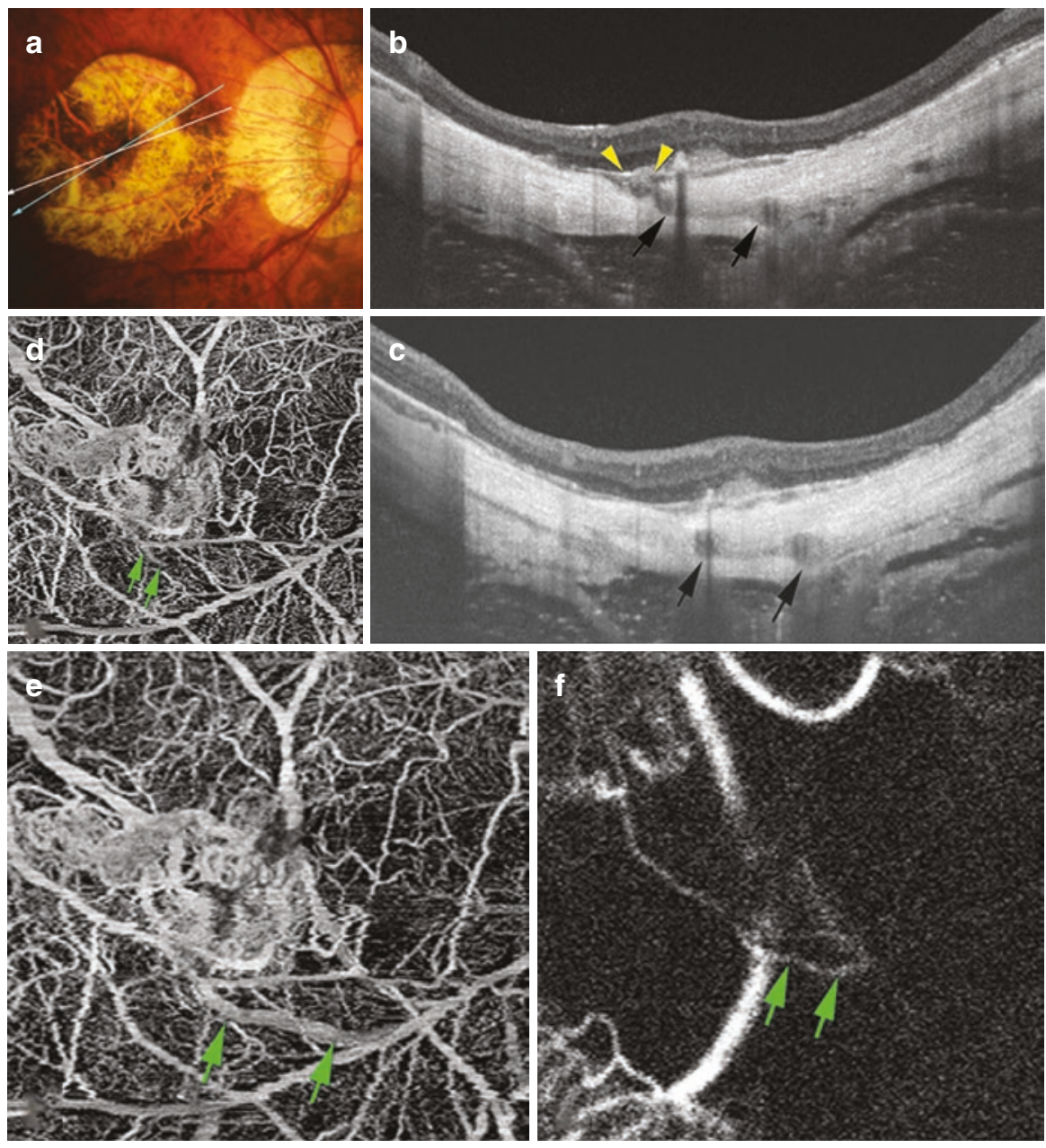

Fig. 9.11 Continuity between scleral perforating vessels and myopic choroidal neovascularization $(\mathrm{CNV})$ in the atrophic phase [38]. (a) Fundus photograph of the right eye shows a sharpmargined macular atrophy around a CNV (arrow). Arrows indicate scanned line by OCT. (b, c) Swept-source OCT images show subretinal CNV. Cross sections of blood vessels coursing within the sclera can be seen (arrows in $\mathbf{c}$ ) in a section scanned by swept-source OCT (blue arrow in $\mathbf{a}$ ). In a serial OCT section (b, in a section scanned at white arrow in a), this vessel (arrows) is observed to be continuous with the CNV through a defect of Bruch's membrane (between arrowheads). (d, e) OCT angiogram shows the intrascleral vessel in Fig. 9.4b begins to be seen at the depth just posterior to the CNV (arrows, $\mathbf{d}$ ). At a deeper slab e, the longer course of this vessel is seen (arrow, e). (f, $\mathbf{g})$ Arterial phase of ICG angiogram ( $6 \mathrm{~s}$ after dye injection in $\mathbf{f}$, and $8 \mathrm{~s}$ after dye injection in g) shows that the intrascleral vessel shown in OCT angiogram and OCT b scans was already filled with dye in the arterial phase (arrows). (h) Arterial phase of fluorescein angiogram (10 s after dye injection) shows that the intrascleral vessel observed in $\mathbf{f}$ and $\mathbf{g}$ is fairly seen (arrows) 

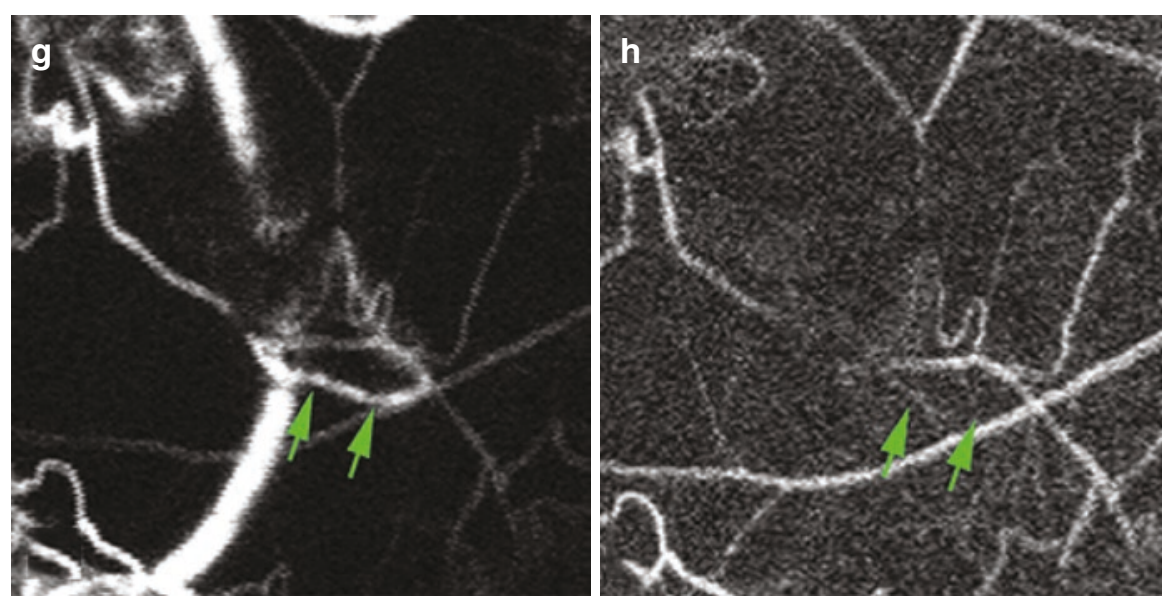

Fig. 9.11 (continued)

also called foveal retinoschisis [39], macular retinoschisis [41], or myopic foveoschisis [42], includes the features of schisis-like inner retinal fluid, schisis-like outer retina fluid, foveal detachment, lamellar or full-thickness macular hole, and/or macular detachment [43]. These features can best be detected by OCT as an indispensable tool to diagnose MTM. Additional examination techniques are a retro-mode imaging which uses an infrared laser in the confocal scanning laser ophthalmoscope and which can produce a pseudo-three-dimensional image showing the details of deep retinal structures (Fig. 9.12). Applying retro-mode imaging, a characteristic fingerprint and firework pattern at the corresponding area of a macular retinoschisis have been detected in the region of a macula retinoschisis [44, 45].

Shimada et al. have classified myopic traction maculopathy according to its location and extent from S0 through S4: S0: no retinoschisis; S1: extrafoveal; S2: foveal; S3: both foveal and extrafoveal but not the entire macula; and S4: entire macula [46].

\subsection{Dome-Shaped Macula (DSM)}

A dome-shaped macula (DSM) is an inward protrusion of the macula as visualized by OCT (Fig. 9.13) [47-49]. Imamura et al. reported that a DSM was associated with, and caused by, a local thickening of the subfoveal sclera [50]. It was postulated that the local thickening of the subfoveal sclera was an adaptive or 

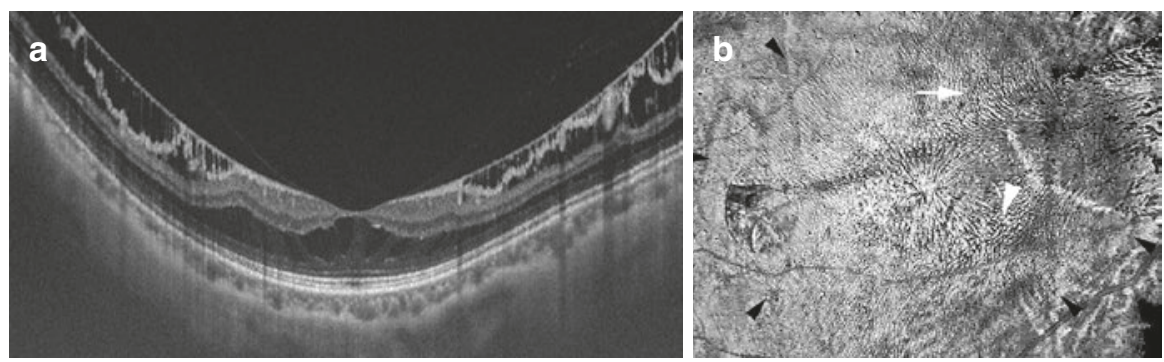

Fig. 9.12 Myopic macular retinoschisis. (a) OCT image shows outer and inner retinoschisis. (b) Columnar structures are seen within the area of outer retinoschisis (cited from [44]). Retro-mode image by F10 (Nidek, Aichi, Japan) showing a fingerprint pattern (black arrowheads) consisting of central radiating retinal striae and surrounding multiple dots (arrowhead) and lines (arrow). Many lines appear in parallel or in a whorled pattern. The inner lamellar hole appears as a circular defect at the central fovea
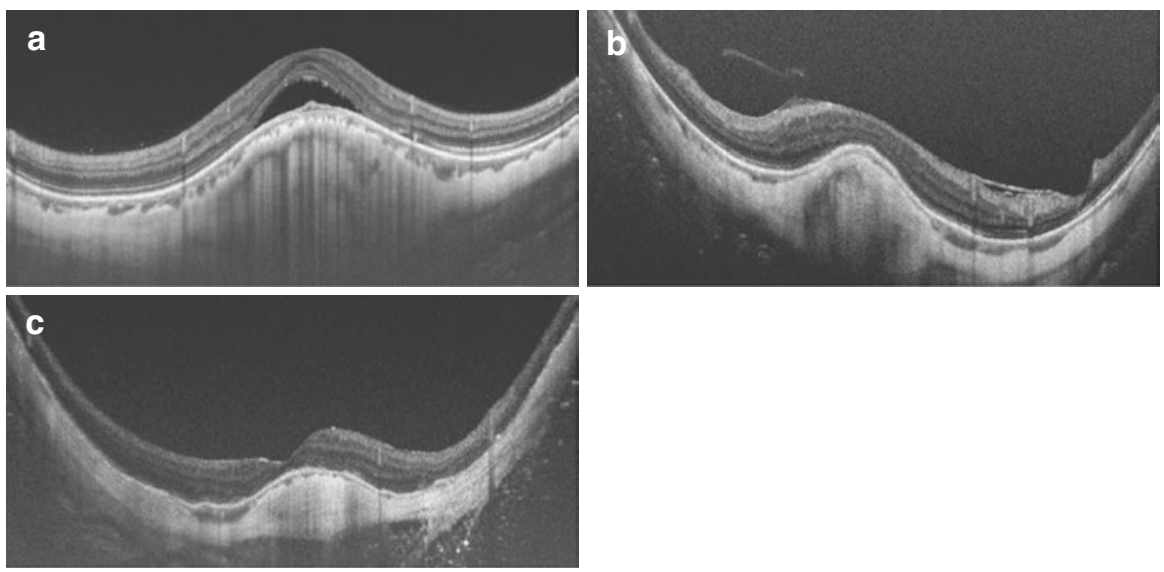

Fig. 9.13 Various types of Dome-shaped macula (DSM). (a) Serous retinal detachment is seen on the top of the dome. The sclera is too thick to allow the visualization of its outer surface. (b) The subfoveal sclera is thick; however, the scleral outer surface is visible in the surrounding area. (c) The subfoveal sclera is thick; however, the scleral outer surface is visible in all regions

compensatory response to the defocus of the image on the fovea in highly myopic eyes. Fang et al. [51] found the high prevalence of macular BM defects around the dome (Figs. 9.14 and 9.15). Ohno-Matsui et al. reported a similar finding [52] as peri-dome choroidal deepening. The morphology of the DSM in association with macular BM defects may be associated with a focal relaxation of the posterior sclera, no longer pushed outward by an expanding BM but allowed to partially bulge inward, leading to the formation of a DSM. 

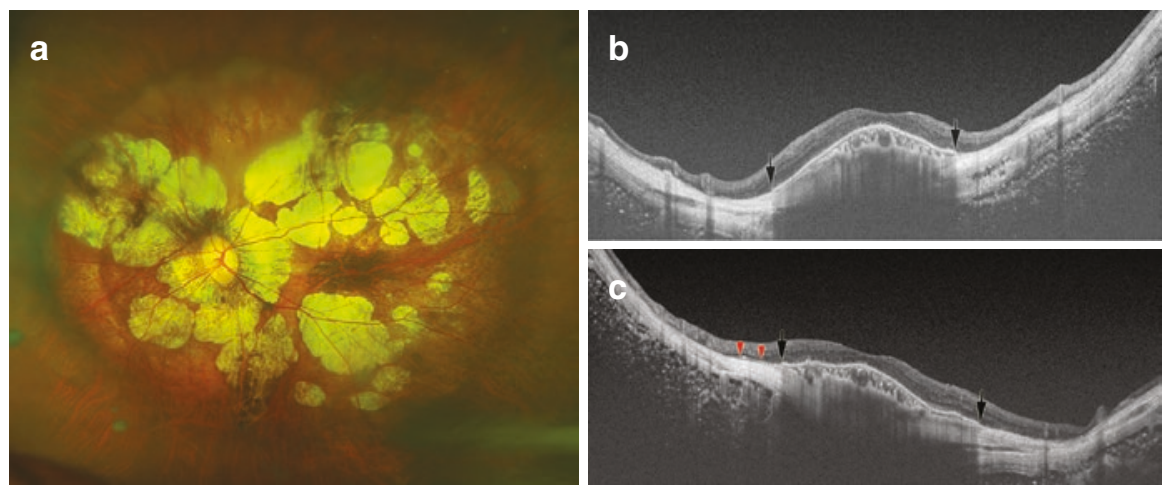

Fig. 9.14 Dome-shaped macula (DSM) coexisting with the macular Bruch's membrane defects (MBMD) associated with patchy atrophy [51]. (a) Left fundus of a 65-year-old woman with an axial length of $33.2 \mathrm{~mm}$ shows multiple patches of whitish, well-defined patchy atrophy in the regions superior and inferior to the fovea. Two long arrows show scanned lines examined by optical coherence tomography (OCT) in the images (b) and (c), respectively. (b) Oblique OCT section across the fovea demonstrates an inward protrusion due to DSM. Bruch's membrane is present at the center of the macula, but is interrupted abruptly (arrow) on both sides of the dome. (c) Oblique OCT section across the fovea shows the inward protrusion of macula due to DSM. Bruch's membrane is present at the center of macula, but is abruptly interrupted (arrow) on both sides of the dome. The remnants of Bruch's membrane are seen near the edge of the macular Bruch's membrane defect (arrowheads)
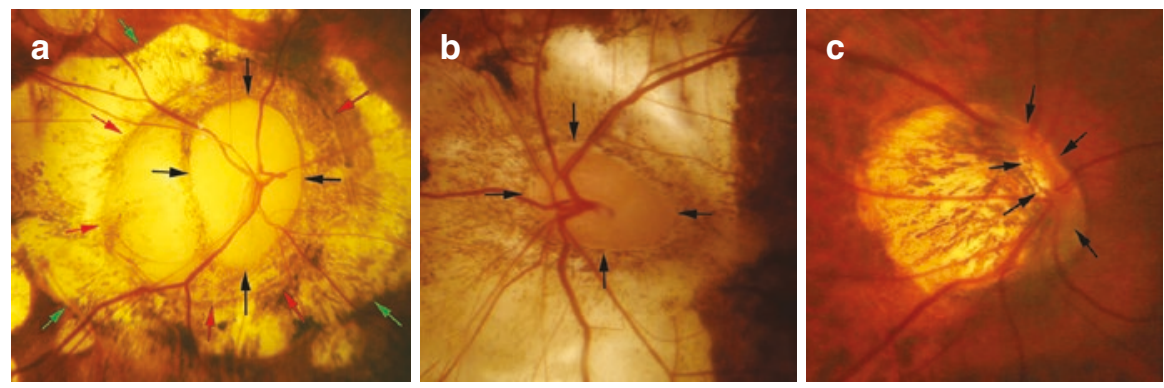

Fig. 9.15 Severe deformity of the optic disc due to pathologic myopia. (a) Acquired megalodisc. The optic disc shows irregular shape. (b) Horizontally stretched optic disc. (c) Extreme tilting of the optic disc. Black arrows: peripapillary rim; red arrows: parapapillary delta zone; green arrows: parapapillary gamma zone

\section{References}

1. Iwase A, Araie M, Tomidokoro A, et al. Prevalence and causes of low vision and blindness in a Japanese adult population: the Tajimi Study. Ophthalmology. 2006;113:1354-62.

2. Xu L, Wang Y, Li Y, et al. Causes of blindness and visual impairment in urban and rural areas in Beijing: the Beijing Eye Study. Ophthalmology. 2006;113:1134.e1. 
3. You QS, Xu L, Yang H, et al. Five-year incidence of visual impairment and blindness in adult Chinese the Beijing Eye Study. Ophthalmology. 2011;118:1069-75.

4. Yamada M, Hiratsuka Y, Roberts CB, et al. Prevalence of visual impairment in the adult Japanese population by cause and severity and future projections. Ophthalmic Epidemiol. 2010;17:50-7.

5. Hsu WM, Cheng CY, Liu JH, et al. Prevalence and causes of visual impairment in an elderly Chinese population in Taiwan: the Shihpai Eye Study. Ophthalmology. 2004;111:62-9.

6. Ohno-Matsui K, Lai TYY, Cheung CMG, Lai CC. Updates of pathologic myopia. Prog Retin Eye Res. 2016;52:156-87.

7. Ohno-Matsui K, Kawasaki R, Jonas JB, et al. International photographic classification and grading system for myopic maculopathy. Am J Ophthalmol. 2015;159:877-83.

8. Fang Y, Yokoi T, Nagaoka N, et al. Progression of myopic maculopathy during 18-year followup. Ophthalmology. 2018;125(6):863-77.

9. Hayashi K, Ohno-Matsui K, Shimada N, et al. Long-term pattern of progression of myopic maculopathy: a natural history study. Ophthalmology. 2010;117:1595-611.

10. Yan YN, Wang YX, Yang Y, et al. Ten-year progression of myopic maculopathy: The Beijing Eye Study 2001-2011. Ophthalmology. 2018;125(8):1253-63.

11. Vongphanit J, Mitchell P, Wang JJ. Prevalence and progression of myopic retinopathy in an older population. Ophthalmology. 2002;109:704-11.

12. Xu L, Wang Y, Wang S, Jonas JB. High myopia and glaucoma susceptibility the Beijing Eye Study. Ophthalmology. 2007;114:216-20.

13. Nagaoka N, Jonas JB, Morohoshi K, et al. Glaucomatous-type optic discs in high myopia. PLoS One. 2015;10:e0138825.

14. Spaide RF. Staphyloma: part 1. New York: Springer; 2014. p. 167-76.

15. Moriyama M, Ohno-Matsui K, Hayashi K, et al. Topographical analyses of shape of eyes with pathologic myopia by high-resolution three dimensional magnetic resonance imaging. Ophthalmology. 2011;118:1626-37.

16. Moriyama M, Ohno-Matsui K, Modegi T, et al. Quantitative analyses of high-resolution 3D MR images of highly myopic eyes to determine their shapes. Invest Ophthalmol Vis Sci. 2012;53:4510-8.

17. Ohno-Matsui K. Proposed classification of posterior staphylomas based on analyses of eye shape by three-dimensional magnetic resonance imaging. Ophthalmology. 2014;121:1798-809.

18. Shinohara K, Shimada N, Moriyama M, et al. Posterior staphylomas in pathologic myopia imaged by widefield optical coherence tomography. Invest Ophthalmol Vis Sci. 2017;58: 3750-8.

19. Shinohara K, Tanaka N, Jonas JB, et al. Ultra-widefield optical coherence tomography to investigate relationships between myopic macular retinoschisis and posterior staphyloma. Ophthalmology. 2018;125(10):1575-86.

20. Curtin BJ. The posterior staphyloma of pathologic myopia. Trans Am Ophthalmol Soc. 1977;75:67-86.

21. Verkicharla PK, Ohno-Matsui K, Saw SM. Current and predicted demographics of high myopia and an update of its associated pathological changes. Ophthalmic Physiol Opt. 2015;35:465-75.

22. Ohno-Matsui K, Jonas JB, Spaide RF. Macular Bruch membrane holes in highly myopic patchy chorioretinal atrophy. Am J Ophthalmol. 2016;166:22-8.

23. Gao LQ, Liu W, Liang YB, et al. Prevalence and characteristics of myopic retinopathy in a rural Chinese adult population: the Handan Eye Study. Arch Ophthalmol. 2011;129:1199-204.

24. Zheng Y, Lavanya R, Wu R, et al. Prevalence and causes of visual impairment and blindness in an urban Indian population: the Singapore Indian Eye Study. Ophthalmology. 2011;118:1798-804.

25. Neelam K, Cheung CM, Ohno-Matsui K, et al. Choroidal neovascularization in pathological myopia. Prog Retin Eye Res. 2012;31:495-525. 
26. Xu X, Fang Y, Uramoto K, et al. Clinical features of lacquer cracks in eyes with pathologic myopia. Retina. 2018. https://doi.org/10.1097/IAE.0000000000002168.

27. Shinohara K, Moriyama M, Shimada N, et al. Myopic stretch lines: linear lesions in fundus of eyes with pathologic myopia that differ from lacquer cracks. Retina. 2014;34:461-9.

28. Sayanagi K, Ikuno Y, Uematsu S, Nishida K. Features of the choriocapillaris in myopic maculopathy identified by optical coherence tomography angiography. Br J Ophthalmol. 2017;101:1524-9.

29. Ohno-Matsui K, Ito M, Tokoro T. Subretinal bleeding without choroidal neovascularization in pathologic myopia. A sign of new lacquer crack formation. Retina. 1996;16:196-202.

30. Shapiro M, Chandra SR. Evolution of lacquer cracks in high myopia. Ann Ophthalmol. 1985;17:231-5.

31. Klein RM, Green S. The development of lacquer cracks in pathologic myopia. Am J Ophthalmol. 1988;106:282-5.

32. Ohno-Matsui K, Yoshida T, Futagami S, et al. Patchy atrophy and lacquer cracks predispose to the development of choroidal neovascularisation in pathological myopia. Br J Ophthalmol. 2003;87:570-3.

33. Wolf S, Balciuniene VJ, Laganovska G, et al. RADIANCE: a randomized controlled study of ranibizumab in patients with choroidal neovascularization secondary to pathologic myopia. Ophthalmology. 2014;121:682-92.

34. Ikuno Y, Ohno-Matsui K, Wong TY, et al. Intravitreal aflibercept injection in patients with myopic choroidal neovascularization: The MYRROR Study. Ophthalmology. 2015;122:1220-7.

35. Ohno-Matsui K, Jonas JB, Spaide RF. Macular Bruch's membrane holes in choroidal neovascularization-related myopic macular atrophy by swept-source optical coherence tomography. Am J Ophthalmol. 2015;162:133-9.

36. Louzada RN, Ferrara D, Novais EA, et al. Analysis of scleral feeder vessel in myopic choroidal neovascularization using optical coherence tomography angiography. Ophthalmic Surg Lasers Imaging Retina. 2016;47:960-4.

37. Giuffre C, Querques L, Carnevali A, et al. Choroidal neovascularization and coincident perforating scleral vessels in pathologic myopia. Eur J Ophthalmol. 2017;27:e39-45.

38. Ishida T, Watanabe T, Yokoi T, et al. Possible connection of short posterior ciliary arteries to choroidal neovascularisations in eyes with pathologic myopia. Br J Ophthalmol. 2018;103(4):457-62.

39. Takano M, Kishi S. Foveal retinoschisis and retinal detachment in severely myopic eyes with posterior staphyloma. Am J Ophthalmol. 1999;128:472-6.

40. Panozzo G, Mercanti A. Optical coherence tomography findings in myopic traction maculopathy. Arch Ophthalmol. 2004;122:1455-60.

41. Benhamou N, Massin P, Haouchine B, et al. Macular retinoschisis in highly myopic eyes. Am J Ophthalmol. 2002;133:794-800.

42. Ikuno Y, Sayanagi K, Ohji M, et al. Vitrectomy and internal limiting membrane peeling for myopic foveoschisis. Am J Ophthalmol. 2004;137:719-24.

43. Johnson MW. Myopic traction maculopathy: pathogenic mechanisms and surgical treatment. Retina. 2012;32(Suppl 2):S205-10.

44. Tanaka Y, Shimada N, Ohno-Matsui K, et al. Retromode retinal imaging of macular retinoschisis in highly myopic eyes. Am J Ophthalmol. 2010;149:635-40.e1.

45. Su Y, Zhang X, Wu K, et al. The noninvasive retro-mode imaging of confocal scanning laser ophthalmoscopy in myopic maculopathy: a prospective observational study. Eye. 2014;28:998-1003.

46. Shimada N, Tanaka Y, Tokoro T, Ohno-Matsui K. Natural course of myopic traction maculopathy and factors associated with progression or resolution. Am J Ophthalmol. 2013;156:948-57.

47. Gaucher D, Erginay A, Lecleire-Collet A, et al. Dome-shaped macula in eyes with myopic posterior staphyloma. Am J Ophthalmol. 2008;145:909-14.

48. Caillaux V, Gaucher D, Gualino V, et al. Morphologic characterization of dome-shaped macula in myopic eyes with serous macular detachment. Am J Ophthalmol. 2013;156:958-67. 
49. Ellabban AA, Tsujikawa A, Matsumoto A, et al. Three-dimensional tomographic features of dome-shaped macula by swept-source optical coherence tomography. Am J Ophthalmol. 2012;3:578.

50. Imamura Y, Iida T, Maruko I, et al. Enhanced depth imaging optical coherence tomography of the sclera in dome-shaped macula. Am J Ophthalmol. 2011;151:297-302.

51. Fang Y, Jonas JB, Yokoi T, et al. Macular Bruch's membrane defect and dome-shaped macula in high myopia. PLoS One. 2017;12:e0178998.

52. Ohno-Matsui K, Fang Y, Uramoto K, et al. Peri-dome choroidal deepening in highly myopic eyes with dome-shaped maculas. Am J Ophthalmol. 2017;183:134-40.

Open Access This chapter is licensed under the terms of the Creative Commons Attribution 4.0 International License (http://creativecommons.org/licenses/by/4.0/), which permits use, sharing, adaptation, distribution and reproduction in any medium or format, as long as you give appropriate credit to the original author(s) and the source, provide a link to the Creative Commons license and indicate if changes were made.

The images or other third party material in this chapter are included in the chapter's Creative Commons license, unless indicated otherwise in a credit line to the material. If material is not included in the chapter's Creative Commons license and your intended use is not permitted by statutory regulation or exceeds the permitted use, you will need to obtain permission directly from the copyright holder.

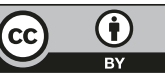

